\title{
CONHECIMENTO DOS PROFISSIONAIS DE ENFERMAGEM DE CENTRO CIRÚRGICO SOBRE HIPOTERMIA EM PACIENTES CIRÚRGICOS ONCOLÓGICOS
}

\author{
Knowledge of the nursing staff in a surgical \\ center about hypothermia in cancer surgical patients \\ Conocimiento de losprofesionales de enfermería de centro \\ quirúrgico sobre hipotermia en pacientes quirúrgicos oncológicos \\ Anderson de Souza', Soraya Palazzo², Débora Montezello ${ }^{3}$
}

RESUMO: Objetivo: Este estudo teve como objetivo analisar o conhecimento dos profissionais de enfermagem de centro cirúrgico sobre hipotermia em pacientes cirúrgicos oncológicos, antes e após intervenção educativa. Método: Trata-se de um estudo descritivo de caráter exploratório, transversal, de campo e com abordagem quantitativa. Foram entrevistados 21 profissionais de enfermagem, e os dados foram coletados antes e após a intervenção educativa. Resultados: O resultado após a aplicação do teste mostrou maior aprovação, e a intervenção educativa evidenciou melhor aproveitamento em comparação ao teste de conhecimento prévio dos profissionais de enfermagem. Conclusão: Diante desse fato, compete ao enfermeiro o planejamento de intervenções e o estabelecimento de prioridades para que metas sejam alcançadas, diminuindo complicações relacionadas à hipotermia. Por fim, é fundamental que o enfermeiro de centro cirúrgico norteie sua equipe e seja o elo do conhecimento técnico-científico para melhorar a qualidade da assistência ao paciente cirúrgico.

Palavras-chave: Enfermagem perioperatória. Hipotermia. Enfermagem em pós-anestésico.

ABSTRACT: Objective: This study aimed to analyze the knowledge of the surgical center nursing staff about hypothermia in oncological surgical patients, before and after educational intervention. Method: This is a descriptive exploratory, cross-sectional, field study with a quantitative approach. Twentyone nursing professionals were interviewed, and data were collected before and after the educational intervention. Results: The result after application of the test showed greater approval, and the educational intervention evidenced better use in comparison with the prior test to assess the knowledge of the nursing professionals. Conclusion: Faced with this fact, it is up to the nurse to plan for interventions and to establish priorities for achieving goals, in order to reduce complications related to hypothermia. Therefore, it is critical that the surgical center nurse guide the team and be the link between technical and scientific knowledge in order to improve the quality of the surgical patient care.

Keywords: Perioperative nursing. Hypothermia. Post-anesthesia nursing.

RESUMEN: Objetivo: Este estudio tiene como objetivo el análisis del conocimiento de los profesionales de la enfermería del cirujano sobre la hipotermia en los pacientes cirúrgicos oncológicos, antes y después de la intervención educativa. Método: Trata-se de un estudio descritivo de caráter exploratorio, transversal, de campo y com abordaje cuantitativo. Foram entrevistados 21 profesionales de la enfermería, y los datos fueron recogidos antes y después de una intervención educativa. Resultados: El resultado de la aplicación de la prueba debe ser mayor, y una intervención educativa es evidente. Conclusión: Diante el hecho, compite en el enfermo o el planeamiento de lasintervenciones y el establecimiento de las mediciones para que se cumplan, disminuyendo las complicaciones relacionadas a la hipotermia. Diantedisso, es fundamental que el enfermero de cirujano centro norteamericano su equipo de medicina o elo de conocimiento técnico-científico para mejorar la calidad de la asistencia al paciente cirúrgico.

Palabras-clave: Enfermería perioperatoria. Hipotermia. Enfermeríaposanestésica.

'Enfermeiro; especialista em Centro Cirúrgico, Recuperação Anestésica e Centro de Material e Esterilização pelo Centro Universitário São Camilo; enfermeiro Júnior do Centro Cirúrgico do AC Camargo Cancer Center - São Paulo (SP), Brasil. E-mail: andersondesouzasp@gmail.com

Rua Professor Antônio Prudente, 211 - Liberdade - CEP: 01509-010 - São Paulo (SP), Brasil.

${ }^{2}$ Enfermeira; mestre pela Universidade de São Paulo (USP); coordenadora dos cursos de pós-graduação Enfermagem em Centro Cirúrgico, Recuperação Anestésica e Centro de Material e Esterilização e Assistência Multidisciplinar em Oncologia do Centro Universitário São Camilo - São Paulo (SP), Brasil.

${ }^{3}$ Enfermeira; mestre em Bioética pelo Centro Universitário São Camilo; diretora de serviços assistenciais do Instituto Brasileiro de Controle do Câncer - São Paulo (SP), Brasil.

Recebido: 23 abr. 2017 - Aprovado: 21 jul. 2017

DOI: $10.5327 / Z 1414-4425201700040003$ 


\section{INTRODUÇÃO}

A hipotermia é responsável por repercussões extremamente deletérias ao organismo, principalmente no período intraoperatório, ou seja, durante as primeiras horas após aplicação da anestesia a temperatura central diminui quase linearmente de 0,5 até $1,5^{\circ} \mathrm{C}$. A anestesia geral contribui para diminuir a temperatura corpórea por meio da inibição direta da termorregulação hipotalâmica, ocasionando complicações sistêmicas como: hiperatividade simpática, com aumento do consumo de oxigênio, vasoconstrição, redução da função plaquetária, diminuição da cascata da coagulação, acarretando o aumento das perdas sanguíneas e a consequente necessidade de transfusão sanguínea ${ }^{1}$.

O paciente cirúrgico necessita que sua temperatura interna seja constante, somente assim as funções metabólicas são conservadas. Considerando-se o exposto, a implementação de métodos para a manutenção da normotermia do paciente no intraoperatório é crucial, e prestar um cuidado competente, qualificado e individualizado é de responsabilidade da equipe multidisciplinar, sendo que cada integrante focando diferentes ângulos a fim de prevenir a hipotermia ${ }^{2,3}$.

Nesse cenário, compete à equipe de enfermagem a implantação de intervenções eficazes que proporcionem a prevenção ou o tratamento da hipotermia e, consequentemente, a diminuição das complicações a ela associadas. Estratégias de aquecimento ativo (sistema de ar forçado aquecido, sistema de circulação de água aquecida) ou passivo (isolantes térmicos, como cobertores, por exemplo) devem ser utilizadas durante o perioperatório para garantir qualidade na assistência ao paciente cirúrgico ${ }^{2,4,5}$.

Portanto, a compreensão sobre a fisiopatogenia, as complicações e as formas de prevenção é importante para que o profissional de enfermagem de centro cirúrgico desempenhe seu papel com competência e seja capaz de utilizar esse conhecimento para assistir ao paciente com qualidade e segurança em situações de hipotermia ${ }^{6}$.

Desse modo, proporcionar programas de educação permanente no centro cirúrgico, que atendam adequadamente às carências dos colaboradores, pode conduzir a mudanças nas atividades desenvolvidas, relacionando teoria e prática em benefício da assistência prestada ${ }^{7}$.

A teoria da aprendizagem significativa propõe um modelo para o processo de assimilação de novas informações na estrutura cognitiva. Assim, a aprendizagem consiste na ampliação dessa estrutura por meio da incorporação de novos conhecimentos, dependendo do tipo de relacionamento que se tem entre as ideias já existentes nessa estrutura e as novas ideias que se internalizam, resultando em um aprendizado que varia do mecânico ao significativo ${ }^{6}$.

Por outro lado, a aprendizagem precisa ser substantiva, isto é, uma vez que determinado conteúdo é aprendido, a pessoa deve ser capaz de explicá-lo com suas próprias palavras. Assim, um mesmo conceito pode ser expresso em linguagem semelhante e transmitir o mesmo significado ${ }^{6}$.

Muitos profissionais de enfermagem de centro cirúrgico têm dificuldade para prestar cuidados a pacientes em hipotermia. Usualmente, a abordagem é feita sob estresse, devido a dificuldades estruturais, financeiras, de recursos humanos, técnico-científicos e de domínio do conhecimento acerca de tal condição. Diante dessa situação, a presente pesquisa adota a seguinte pergunta de estudo: qual o conhecimento dos profissionais de enfermagem de centro cirúrgico sobre hipotermia?

\section{OBJETIVO}

Este estudo teve como objetivo analisar o conhecimento dos profissionais de enfermagem de centro cirúrgico sobre hipotermia em pacientes cirúrgicos oncológicos, antes e após intervenção educativa.

\section{MÉTODO}

Trata-se de um estudo descritivo de caráter exploratório, transversal, de campo e com abordagem quantitativa.

A população do estudo foi composta por profissionais de enfermagem que atuavam diretamente na assistência, ou seja, enfermeiros e técnicos de enfermagem. A amostra contou com 21 profissionais de enfermagem, sendo 3 enfermeiros e 18 técnicos de enfermagem.

Utilizaram-se os seguintes critérios para a inclusão dos sujeitos: vínculo empregatício, tempo de atuação superior a um ano na instituição sede da pesquisa e ter aceitado participar da pesquisa mediante assinatura do Termo de Consentimento Livre e Esclarecido (TCLE). Para os critérios de exclusão, consideraram-se os profissionais em férias ou de licença médica.

Trate-se de um hospital privado e filantrópico de grande porte sediado na zona leste do município de São Paulo (SP), Brasil. O centro cirúrgico é constituído de 10 salas cirúrgicas e 10 leitos de recuperação anestésica, sendo realizadas, em média, 450 cirurgias por mês.

Para a coleta de dados, foi utilizado um instrumento elaborado pelos autores, composto por duas partes, sendo a primeira 
a caracterização da amostra (gênero, idade, categoria profissional, tempo de formação e tempo de atuação). A segunda parte consistia no teste de avaliação de conhecimento, contendo oito questões sobre hipotermia, sendo aplicado na fase pré-intervenção e reaplicado um mês após a intervenção educativa.

A realização do teste de conhecimento visou analisar o nível de conhecimento dos profissionais de enfermagem sobre a hipotermia. Os temas abordados foram: definição da hipotermia, fisiopatologia da hipotermia, controle de temperatura, mecanismos de perda de calor, mecanismos de aquecimento ativo e passivo e complicações decorrentes da hipotermia.

Em relação à análise dos dados, foi atribuída para cada item uma pontuação de 1,25 . O escore total do teste de conhecimento correspondeu à soma de todas as respostas corretas. Utilizou-se estatística descritiva, sendo as frequências absolutas e relativas apresentadas por meio de números e porcentagens.

A coleta de dados foi iniciada após a aprovação do Comitê de Ética em Pesquisa (CEP) do Instituto Brasileiro de Controle do Câncer (IBCC) (CAAE n ${ }^{\circ}$ 49630215.1.0000.0072), respeitando os preceitos éticos de pesquisa com seres humanos, fundamentados na Resolução n ${ }^{\circ}$ 466/ 12 do Conselho Nacional de Saúde. Cada profissional elegível foi convidado a participar do estudo, sendo-lhe entregue o TCLE, explicado e assinado pelo pesquisador e por cada participante. Os participantes responderam ao instrumento proposto individualmente, durante a jornada de trabalho, e devolveram-no imediatamente ao pesquisador.

\section{RESULTADOS}

O presente estudo foi composto por 3 enfermeiros e 18 técnicos de enfermagem de centro cirúrgico, os quais foram avaliados quanto ao conhecimento referente à hipotermia em pacientes cirúrgicos oncológicos. A amostra foi composta por toda a equipe do centro cirúrgico, pois não havia colaboradores que preenchiam os critérios de exclusão.

A Tabela 1 contempla os dados sociodemográficos e acadêmicos dos 21 profissionais de enfermagem participantes, dos quais $15(71,4 \%)$ eram do sexo feminino. A faixa etária variou de 20 a 50 anos, em relação ao tempo de formação variou de 1 a 5 anos (47,6\%) e no tempo de atuação, de 1 a 5 anos (52,3\%). Referente à categoria profissional, observou-se o predomínio de técnicos de enfermagem, com 18 participantes (85,71\%).

A Tabela 2 representa as variáveis estudadas, com o intuito de analisar o antes e após a intervenção educativa, referente ao conhecimento dos profissionais de enfermagem sobre hipotermia.
Tabela 1. Caracterização dos profissionais de enfermagem participantes da intervenção educativa, segundo variáveis sociodemográficas e acadêmicas. São Paulo (SP), Brasil, 2016.

\begin{tabular}{|l|c|c|}
\hline Variáveis & $\mathbf{n}$ & $\%$ \\
\hline Sexo & & \\
\hline Masculino & 6 & 28,5 \\
\hline $\begin{array}{l}\text { Feminino } \\
\text { Faixa etária (anos) }\end{array}$ & 15 & 71,4 \\
\hline $20-30$ & 7 & 33,3 \\
\hline $30-40$ & 10 & 47,6 \\
\hline $40-50$ & 4 & 19,0 \\
\hline Categoria profissional & & \\
\hline Enfermeiros & 3 & 14,3 \\
\hline Técnicos de enfermagem & 18 & 85,7 \\
\hline Tempo de formação (anos) & & \\
\hline $1-5$ & 10 & 47,6 \\
\hline $5-10$ & 6 & 28,5 \\
\hline $10-15$ & 5 & 23,8 \\
\hline Tempo de atuação (anos) & & \\
\hline $1-5$ & 11 & 52,3 \\
\hline $5-10$ & 8 & 38,0 \\
\hline $10-15$ & 2 & 9,5 \\
\hline
\end{tabular}

Fonte: dados da pesquisa.

Tabela 2. Pontuação dos profissionais de enfermagem participantes em questões sobre o conhecimento de hipotermia, segundo os itens de avaliação antes e após a intervenção educativa - São Paulo (SP), Brasil, 2016.

\begin{tabular}{|l|c|c|c|}
\hline Variáveis & $\begin{array}{c}\text { Antes } \\
\mathbf{n}(\%)\end{array}$ & $\begin{array}{c}\text { Após } \\
\mathbf{n}(\%)\end{array}$ & $\begin{array}{c}\text { Diferença } \\
\mathbf{n}(\%)\end{array}$ \\
\hline $\begin{array}{l}\text { Conceito sobre hipotermia } \\
\text { Centro termorregulador }\end{array}$ & $13(61,9)$ & $18(85,7)$ & $5(23,8)$ \\
\hline $\begin{array}{l}\text { Sinais de hipotermia } \\
\text { Consequências fisiológicas } \\
\text { no organismo }\end{array}$ & $5(23,8)$ & $16(76,1)$ & $11(52,3)$ \\
\hline $\begin{array}{l}\text { Mecanismo de perda de calor } \\
\text { Avaliação da temperatura }\end{array}$ & $11(52,3)$ & $13(61,9)$ & $6(28,6)$ \\
\hline $\begin{array}{l}\text { Métodos e medidas } \\
\text { preventivas de aquecimento }\end{array}$ & $13(61,9)$ & $18(85,6)$ & $13(61,8)$ \\
\hline $\begin{array}{l}\text { Fatores que contribuem para } \\
\text { a hipotermia }\end{array}$ & $14(66,6)$ & $19(90,4)$ & $5(23,4)$ \\
\hline
\end{tabular}




\section{DISCUSSÃO}

Os resultados evidenciaram que, das 8 questões respondidas antes da intervenção educativa, 2 apresentaram baixo índice de acerto, sendo-as: sinais de hipotermia, com 5 respostas corretas (23,8\%), e consequências fisiológicas no organismo, com 7 respostas corretas (33,3\%). Após a intervenção educativa, as mesmas questões apresentaram $16(76,1 \%)$ e $13(61,9 \%)$ respostas corretas, respectivamente.

Do restante das questões, 6 apresentaram índice de acerto de 52,3 a $66,6 \%$, as quais abordam o conhecimento sobre: centro termorregulador, mecanismos de perda de calor, avaliação da temperatura, medidas e métodos preventivos de aquecimento e fatores que contribuem para a hipotermia. Depois da intervenção educativa, todas as questões apresentaram aumento no índice de acertos, entretanto o item referente aos mecanismos de perda de calor teve alta de apenas $1,27 \%$. Três questões apresentaram 18 respostas corretas $(85,7 \%)$, e apenas uma chegou a $19(90,4 \%)$.

Desde modo, é importante a identificação de fatores de risco no período intraoperatório que, isoladamente ou em conjunto, possam ser controlados para amenizar as complicações da hipotermia em pacientes submetidos a procedimento anestésico-cirúrgico ${ }^{8}$.

A prevenção da hipotermia se mostra relevante, sobretudo, em pacientes submetidos a cirurgias de médio e grande porte. Assim, há necessidade de intervenções para a prevenção da hipotermia em pacientes cirúrgicos e a manutenção da normotermia ${ }^{9,10}$.

A manutenção da temperatura é um aspecto importante para a segurança do paciente e a obtenção de resultados cirúrgicos positivos, como: diminuição do sangramento; redução de infecção do sítio cirúrgico; menor tempo de permanência na sala de recuperação anestésica; aumento do conforto térmico; satisfação do paciente e por fim redução dos custos hospitalares ${ }^{11,12}$.

Como medidas para prevenir a hipotermia, devem ser empregadas estratégias de aquecimento passivo ou ativo. O aquecimento passivo é um método eficaz de baixo custo que consiste em cobrir, durante o período perioperatório, toda a superfície cutânea possível do paciente com lençóis ou cobertores. Essa estratégia pode reduzir a perda de calor em até $30 \%$. O aquecimento ativo por sistema de ar forçado aquecido é um método eficiente para a prevenção da hipotermia em pacientes cirúrgicos ${ }^{13,14}$.
$\mathrm{O}$ aquecimento insuficiente pode resultar na diminuição da temperatura do corpo em 2 a $6^{\circ} \mathrm{C}$, ocasionando complicações cirúrgicas. Assim, é essencial o uso de isolamento e dispositivos de aquecimento no perioperatório. Na prática clínica, o isolamento passivo e a ativação de sistemas de aquecimento têm sido frequentemente usados para prevenir a hipotermia durante o procedimento anestésico-cirúrgico ${ }^{15}$.

A combinação de dispositivos de aquecimento ativo por ar forçado com colchão térmico é geralmente usada para aquecer os pacientes durante a cirurgia, mas não há evidências suficientes que comprovem a necessidade dessa associação. Segundo as diretrizes do National Institute for Health and Clinical Excellence, o dispositivo de aquecimento por ar forçado é a principal medida de prevenção contra a hipotermia ${ }^{16}$.

Os resultados elucidam a importância de intervenções educativas sobre o conhecimento em hipotermia dos profissionais de enfermagem. Isso significa que é fundamental para o enfermeiro de centro cirúrgico nortear sua equipe e ser o elo do conhecimento técnico-científico a fim de melhorar a qualidade da assistência ao paciente cirúrgico. Desse modo, os processos de qualificação dos profissionais de enfermagem devem ser estruturados com base na problemática do seu processo de trabalho.

A formação dos profissionais de enfermagem exige continuidade e educação permanente, sendo progressiva e transdisciplinar. Com isso, é capaz de propiciar a democratização institucional, o desenvolvimento das capacidades de aprendizagem e de criatividade para as situações de saúde, o trabalho em equipe matricial e a melhora de forma permanente a qualidade de saúde, bem como práticas críticas, éticas e humanísticas ${ }^{17}$.

\section{CONCLUSÃO}

A hipotermia durante o período intraoperatório é comum e ocorre como resultado de uma desordem da termorregulação induzida pela anestesia, devido ao ato cirúrgico e ao ambiente. Portanto, é necessário capacitar os profissionais de enfermagem de centro cirúrgico para atuarem em situações adversas.

O presente estudo demonstrou a necessidade da elaboração de programas educativos e de um protocolo de cuidado direcionado para a prevenção ou o tratamento da hipotermia no período perioperatório. Tal prática deve ser estendida a toda a equipe, sendo esse um processo amplo que confere, sobretudo, compromisso social e credibilidade ao serviço prestado pela enfermagem ao paciente cirúrgico. Assim, tal 
procedimento é necessário para a organização e o crescimento da equipe de enfermagem, além de representar um instrumento para a melhoria da qualidade da assistência. Diante desse fato, compete ao enfermeiro o planejamento de intervenções e o estabelecimento de prioridades para que metas sejam alcançadas, diminuindo a chance de complicações relacionadas à hipotermia.
O estudo teve como limitação o baixo número de profissionais de enfermagem, principalmente no que tange à amostra de enfermeiros, que correspondeu a três profissionais. Têm-se como perspectiva novos estudos em hipotermia que possam contribuir para o estímulo à aquisição de conhecimento por parte dos profissionais de enfermagem.

\section{REFERÊNCIAS}

1. Kurnat-Thoma EL, Roberts MM, Corcoran EB. Perioperative Heat Loss Prevention-A Feasibility Trial. AORN J. 2016 Oct;104(4):30719. doi: 10.1016/j.aorn.2016.07.012

2. Poveda VB, Galvão CM. Hipotermia no período intra-operatório: é possível evitá-la? Rev Esc Enferm USP [Internet]. 2011 [acesso em 2015 set. 10];45(2):411-7. Disponivel em: http://www.scielo.br/pdf/ reeusp/v45n2/v45n2a15.pdf

3. Moysés AM, Trettene AS, Navarro LHC, Ayres JA. Prevenção da hipotermia no transoperatório:comparação entre manta e colchão térmicos. Rev Esc Enferm USP. 2014;48(2):228-35. http://dx.doi. org/10.1590/S0080-623420140000200005

4. Mattia AL, Barbosa MH, Rocha AM, Pereira NHC. Hipotermia em pacientes na recuperação pós-anestésica: análise da intervenção de infusão venosa aquecida. Rev Eletr Enf. 2014;16(4):787-94. http:// dx.doi.org/10.5216/ree.v16i4.24222

5. Tramontini CC, Graziano KU. Controle da hipotermia de pacientes cirúrgicos idosos no intraoperatório: Avaliação de duas intervenções de enfermagem. Rev Latino-Am Enferm [Internet]. 2007 [acesso em 2015 set. 12];15(4):626-31. Disponível em: http://www.scielo.br/pdf/ rlae/v15n4/pt_v15n4a16.pdf

6. Mendoza IYQ, Peniche ACG, Puschel VAA. Conhecimento sobre hipotermia dos profissionais de Enfermagem do Centro Cirúrgico. Rev Esc Enferm USP [Internet]. 2012 [acesso em 2015 out. 04];46(Esp):123-9. Disponível em: http://www.scielo.br/pdf/ reeusp/v46nspe/18.pdf

7. Mendoza IYQ, Peniche ACG. Intervenção educativa sobre hipotermia: uma estratégia de ensino para aprendizagem em Centro Cirúrgico. Rev Esc Enferm USP. 2012;46(4):851-7. http://dx.doi.org/10.1590/ S0080-62342012000400010

8. Castro FSF, Peniche ACG, Mendoza IYQ, Couto AT. Temperatura corporal, Índice Aldrete e Kroulik e alta do paciente da Unidade de Recuperação Anestésica Pós-Anestésica. Rev Esc Enferm USP [Internet]. 2012 [acesso em 2015 out. 05];46(4):872-6. Disponível em: http://www.scielo.br/pdf/reeusp/v46n4/13.pdf

9. Panossian C, Simões CM, Milani WRO, Baranauskas MB, Margarido CBO. O Uso de Manta Térmica no Intra-Operatório de Pacientes
Submetidos à Prostatectomia Radical Está Relacionado com a Diminuição do Tempo de Recuperação Pós-Anestésica. Rev Bras Anestesiol. 2008;58(3):220-6. http://dx.doi.org/10.1590/ S0034-70942008000300004

10. Pereira NHC, Rocha AM, Mattia AL. Infusão venosa aquecida relacionada à prevenção das complicações da hipotermia intraoperatória. Rev SOBECC. 2014;19(2):74-8. http://dx.doi.org/10.4322/sobecc.2014.013

11. Poveda VB, Galvão CM, Santos CB. Fatores relacionados ao desenvolvimento de hipotermia no período intra-operatório. Rev Latino-am Enferm [Internet]. 2009 [acesso em 2015 ago. 23];1 1(2). Disponível em: http://www.scielo.br/pdf/rlae/v17n2/pt_14.pdf

12. Danczuk RFT, Nascimento ERP, Silveira NR, Hermida PMV, Rasia MA. Métodos de aquecimento na prevenção da hipotermia no intraoperatório de cirurgia abdominal eletiva. Esc Anna Nery. 2015;19(4):578-84. http://dx.doi.org/10.5935/1414-8145.20150077

13. Poveda VB, Martinez EZ, Galvão CM. Métodos ativos de aquecimento cutâneo para prevenção de hipotermia no período intraoperatório: revisão sistemática. Rev. Latino-Am Enferm [Internet]. 2012 [acesso em 2015 ago. 23];20(1):[9 telas]. Disponível em: http://www.scielo. $\mathrm{br} / \mathrm{pdf} / \mathrm{rlae} / \mathrm{v} 20 \mathrm{n} 1 / \mathrm{pt} \_24 . \mathrm{pdf}$

14. Mattia AL, Barbosa MH, Filho JPAF, Rocha AM, Pereira NHC. Infusão venosa aquecida no controle da hipotermia no período intraoperatório. Rev Latino-Am Enferm [Internet]. 2013 [acesso em 2015 out. 02];21(3):[8 telas]. Disponível em: http://www.scielo.br/pdf/rlae/ v21n3/pt_0104-1169-rlae-21-03-0803.pdf

15. Nieh HC, SU SF. Meta-analysis: effectiveness of forced-air warming for prevention of perioperative hypothermia in surgical patients. $J$ Adv Nurs. 2016;72(10):2294-314. doi: 10.1111/jan.13010

16. Al-Qahtani AS, Messahel FM. Benchmarking inadvertent perioperative hypothermia guidelines with the National Institute for Health and Clinical Excellence. Saudi Med J [Internet]. 2011 [acesso em 2011 jul. 11];32(1):27-31. Disponível em: https://www.ncbi.nlm.nih.gov/ pubmed/21212912

17. Ribeiro DR, Longo ART. Hipotermia como fator de risco para infecção de sítio cirúrgico: conhecimento dos profissionais de enfermagem de nível médio. Rev Min Enferm. 2011;15(1):34-41. 